\title{
If someone cares about you, you are more apt to come around: improving HIV care engagement by strengthening the patient-provider relationship
}

This article was published in the following Dove Press journal:

Patient Preference and Adherence

\author{
Troy J Wood' \\ Kimberly A Koester ${ }^{2}$ \\ Katerina A Christopoulos ${ }^{3}$ \\ John A Sauceda' \\ Torsten B Neilands' \\ Mallory O Johnson' \\ 'Department of Medicine, University \\ of California, San Francisco, CA, \\ USA $;{ }^{2}$ AIDS Policy Research Center, \\ Department of Medicine, University \\ of California, San Francisco, CA, \\ USA; ${ }^{3}$ Zuckerberg San Francisco \\ General Hospital, Division of HIV, ID \\ and Global Medicine, University of \\ California, San Francisco, CA, USA
}

Correspondence: Troy J Wood Department of Medicine, University of California, San Francisco, UCSF Box 0886, 550 16th Street, 3rd Floor, San Francisco, CA 94143, USA

Tel +l 4I5 4766402

Email troy.wood@ucsf.edu
Purpose: The patient-provider relationship is a central factor that can promote or hinder longterm engagement in care among people living with chronic illnesses. In this paper, we explore characteristics of the patient-provider relationship that facilitated or hindered engagement in care among patients receiving care at HIV specialty clinics.

Patients and methods: We conducted 6 focus group discussions with a total of 43 wellretained and less well-retained HIV+ patients in San Francisco, Seattle, and Birmingham, to elicit a wide range of perspectives on engagement in HIV care. Borrowing from the field of psychotherapy, we examined patient-provider relationship characteristics through the lens of the therapeutic alliance, and with regard to their therapeutic efficacy and impact on patient engagement.

Results: The majority of participants emphasized how a strong patient-provider relationship defined by trust, intimacy, and collaboration promoted engagement, while a weak patientprovider relationship impeded engagement.

Conclusion: We discuss practical strategies and therapeutic techniques that may be helpful to providers in building strong patient-provider relationship and contend that a strong patientprovider relationship is crucial for patients to feel cared for during clinical encounters, which can promote long-term and sustained engagement in HIV care.

Keywords: focus groups, engagement in care, therapeutic alliance, psychotherapy, HIV, HIV care

\section{Introduction}

The patient-provider relationship is a central factor that can promote or hinder longterm engagement in care among people living with chronic illnesses. ${ }^{1-6}$ Indeed, for HIV-infected individuals, a weak patient-provider relationship can be a significant barrier to linkage and retention in care. ${ }^{4,7}$ In contrast, a strong relationship, as indicated by providers treating patients with respect and dignity through actively listening and offering easy-to-understand explanations, is associated with increased appointment attendance in HIV care settings. ${ }^{4,8}$

In the field of psychotherapy, the "therapeutic alliance" has been defined in various ways. ${ }^{9}$ In general, these definitions converge in depicting the alliance as a collaborative attachment bond between the therapist and the patient through which therapeutic interventions effect beneficial changes in the patient. In a recent concept analysis of 722 articles on engagement in care, the therapeutic alliance emerged as 1 of 4 defining attributes of patient engagement. ${ }^{10}$ Research on the therapeutic alliance parallels much of the research on the patient-provider relationship and supports the conclusion that a weak alliance is a barrier to engagement in mental health services. In studies of 
psychiatric patients undergoing treatment, a weak therapeutic alliance was a major contributor to premature termination of treatment. ${ }^{11-13}$

In addition, the therapeutic alliance can be the most curative factor in psychotherapy. In one meta-analysis, having a strong therapeutic alliance had the greatest impact on positive psychotherapy outcomes, regardless of the theoretical orientation of the therapist. ${ }^{14}$ In another meta-analysis, patient and observer perceptions of the psychotherapist's level of empathy yielded the largest effect size with regard to patient improvement. ${ }^{15}$ As a result, psychotherapists receive extensive training on building and maintaining strong relationships with patients, while medical providers may only receive training on how to communicate effectively, which is one central dimension of a good working relationship. ${ }^{6,16}$

While an enduring and trusting patient-provider relationship is a key factor in the health of people living with HIV (PLWH), ${ }^{17,18}$ the role and responsibility of the provider in building and maintaining a good relationship with the patient has been relatively understudied. Given that the therapeutic alliance is known to account for a substantial proportion of positive outcomes in psychotherapy, an examination of the patient-provider relationship in HIV care through the lens of the therapeutic alliance has the potential to add important insights to the literature on HIV care engagement.

\section{Patients and methods}

As part of a larger study on engagement in HIV care, we conducted six focus groups from February to March of 2014 in San Francisco, Seattle, and Birmingham, with the goal of eliciting perspectives on engagement in care from PLWH. The larger study was funded by the National Institute of Mental Health and is fully described elsewhere. ${ }^{19}$ Briefly, the goal of the larger study was to develop a patient-centered measure of engagement in HIV care using input from a range of perspectives, including HIV and non-HIV researchers, providers and PLWH. Ethical approval was obtained from the institutional review boards at the University of California, San Francisco, the University of Washington, and the University of Alabama at Birmingham.

The patient-provider relationship was one among several factors that emerged as being of key import to engagement, with many participants describing the patient-provider relationship in ways that strongly corresponded with the therapeutic alliance in psychotherapy. Thus, we decided to use the therapeutic alliance as the lens through which to analyze patient-provider relationship factors that facilitated or hindered engagement in care among PLWH. We further discuss the therapeutic utility of these factors and provide recommendations on incorporating key concepts to strengthen rapport between patients and providers in HIV care settings.

The facilitators had extensive experience conducting qualitative research while working with PLWH, including expertise in focus group facilitation. Each group was led by a pair of expert qualitative researchers (and in all but one group at least one $\mathrm{PhD}$ level anthropologist) serving as co-facilitators. A third study team member observed the focus groups and took detailed notes. The focus groups were digitally recorded and transcribed verbatim.

\section{Eligibility}

We purposefully recruited well-retained and less wellretained patients to allow for a wide range of perspectives on engagement in care. Inclusion criteria were: 1) receiving HIV care at 1 of 3 university-affiliated HIV clinics in Seattle, San Francisco, or Birmingham; 2) 18+ years old (19+ for Birmingham participants); and 3) being diagnosed with HIV and in clinic care for at least 12 months. For 1 of the 2 focus groups at each site, we sought to recruit less well-retained patients with the following additional eligibility criteria: having 2 or more primary care appointments no-shows in the past 12 months OR failing to meet the Health Resources and Services Administration's HIV/AIDS Bureau's (HRSA/ $\mathrm{HAB}$ ) definition of engagement in care (at least 2 primary care visits at least 90 days apart in the last 12 months). For the well-retained focus group at each site, additional criteria included having zero no-show visits in the past year, fulfillment of HRSA/HAB measure and currently having an undetectable HIV viral load. ${ }^{20,21}$ The 3 university-affiliated HIV clinics are part of the Center for AIDS Research (CFAR) Network of Integrated Clinical Systems (CNICS).

\section{Recruitment}

Participants were identified and approached in the following ways: 1) CNICS staff identified potential participants as they were completing a standardized patient-reported outcomes survey (administered to CNICS patients every 4-6 months) and invited them to be screened; and 2) providers were contacted via email and at staff meetings and encouraged to refer well-retained and less well-retained patients. The CNICS staff at each site confirmed all eligibility criteria through medical chart reviews after potentially eligible patients were identified. 


\section{Procedures}

After obtaining written informed consent, the facilitators used a semi-structured guide to elicit patients' perspectives on how to define the dimensions of engagement in care. Sample questions included "What comes to mind when you think of a patient who is well-engaged in HIV health care?" and "What role does your provider play in your engagement in care?" and "What role does the clinic play in in your engagement in care?" Each focus group session lasted $\sim 2.5$ hours and concluded with a brief demographic survey. Participants were paid $\$ 50$ in cash for their time and travel, and light refreshments were provided. After each focus group, the facilitators met to discuss overall impressions and wrote field notes detailing emerging themes and participant dynamics.

\section{Data analysis}

The data were coded in an iterative process by 2 analysts using a modified version of directive content analysis. ${ }^{22}$ The primary analyst read the transcripts and identified sections related to the patient-provider relationship. The primary analyst wrote memos to organize and code the data into major a priori concepts (eg, authenticity, acceptance, collaboration) common to the therapeutic alliance, while also analyzing the data for emergent themes (eg, medical mistrust, physical affection). The analysts met regularly to review the memos, allowing the secondary analyst an opportunity to provide input and confirm or refine identified themes. Discrepancies were resolved by reviewing relevant portions of the transcripts together. The primary analyst created a coding template based on these discussions to determine common and divergent themes across the focus groups. The primary analyst presented his findings to the research team at least monthly for feedback. We note that we did not formally set out to compare the data between the well-retained and less well-retained groups; rather, we used those recruitment categories to ensure a wide range of engagement in care backgrounds. While we were cognizant that the provider-patient relationship might differ between groups, we noted evidence of similarities far more frequently between the groups than differences. Thus, we de-emphasized the distinctions between the well-retained and less well-retained patients.

\section{Results}

Forty-three patients participated in the focus groups. The median patient age was 50 years and the majority of participants were men (53\%) and white (51\%). Most non-white patients were African-American (37\%). Forty-seven percent of the patients self-identified as homosexual (lesbian, gay, or homosexual) and $42 \%$ identified as heterosexual. The patients had been living with an HIV diagnosis for an average of 19 years.

\section{No experience forging relationships with medical providers: "Trust is something that takes time to build"}

Most participants reported having little to no involvement with health care prior to HIV diagnosis. When asked what health and health care meant to one male participant prior to his HIV diagnosis, he remarked that "they were just words" and "didn't exist." Another participant described these terms as "irrelevant" before he was diagnosed with HIV [Site B].

For some, the lack of experience with the health care system included not knowing how to build and maintain a relationship with a provider. A participant explained:

I didn't have a relationship with a doctor, and didn't know how to form one before HIV. Now, it's a whole new ballgame. It's building relationship with that doctor. I don't think they can help you unless you're totally honest with what's going on with you. And trust is something that takes time to build. [Site B]

The quote above highlights the bidirectional nature of the patient-provider relationship with regard to building trust. Patients potentially take a risk when they are honest with their provider, particularly if discussing stigmatized behaviors such as drug use or nonadherence to medications.

Some participants described holding negative views about the medical establishment prior to being diagnosed with HIV: "I just felt like doctors was quacks." [Site C]. Other participants were mistrustful due to stories of family or community members' negative health care experiences. In the words of one participant:

The health care industry has been doing a lot of trial and error over the years, and the patients seem to be the one put in the line of fire most of the time. [Site B]

Another participant approached the subject of trust as something that was "earned" when a provider was willing to step "outside their comfort zone and help [her] understand" what they were saying. She explained the consequences of her mistrust in a provider who was unable or unwilling to do this:

I'm one of these person that you're gonna have to earn my trust before I believe anything that's comin' out of your 
mouth. So if you didn't earn my trust, all your shit that's comin' out your mouth sounds like nothing [...] it can go in one ear and out the other. [Site A]

This kind of skepticism is critical to bear in mind when considering what it takes for a provider to build strong relationships with patients.

Participants with other chronic illnesses found it easier to build strong relationships with providers due to having extensive experience with health care prior to HIV diagnosis:

I was born with hemophilia [...] But because I was born with it, we are very intimate with our health-care providers from day one. So for me, I have been with my health care practitioners all my life. [Site B]

\section{Intimate relationships: "If someone cares, you're more apt to come around them."}

Participants expressed a desire to have a strong working relationship with their providers. For one participant, health care meant "having a good rapport with [my] doctor." [Site A]. When asked how important his relationship with his provider was, another participant responded, "It's very important. Like me, if I don't like you, I don't wanna see you no more." [Site C]. These quotes suggest that the provider's approach at the beginning of the relationship may be crucial to establishing a foundation for a secure relationship.

Participants explained that having a provider who genuinely cared about them was important to helping them stay engaged with one stating, "If someone cares about you, you're more apt to come around them." [Site C]. When asked how to recognize genuine care, several participants noted that the tone of the provider and staff voices mattered, if the staff knew the participants by their names, and if they were physically affectionate (ie, embracing patients in a hug).

Similarly, several participants were appreciative that their providers and staff expressed a sincere interest in them as people and not simply as patients:

I know that every time I walk off the elevator - either the nurses or the doctors, every single one of them on that floor will know me, know my name, they always speak to me. Pass me by - hey, X! And they mean it. They don't just say it. [Site A]

I mean, for me I can just tell, I can feel it. [My doctor] asks about my mom, we're cool like that. She got compassion for me. And that's big for me. [Site A]

Sometimes being known by name was taken as a sign of respect as one participant explained: "they respect you and call you by name. They don't say, hey, come here, like the last place I was at" to which another participant added, "Your name is not 'next'." [Site C]

Participants also discussed the importance of acceptance from their providers. One participant summed it up this way, "[HIV] is just a condition I have, it's not who I am. And it's just the way she just talks to me. She knows me." [Site A]. For another participant, her provider demonstrated acceptance through encouragement and physical touch:

Some clinics you could go to and people treat you so nasty.

They know you got the virus, and they don't want to touch

you, they don't want to be around you. But I could come here

every day if I had to, because I feel the love here. [Site C]

As the above quotes suggest, some patients may be sensitive to how their providers and the clinic staff behave around them. Another participant also commented on the importance of human touch, "I don't really recall a time that I've come in here and they haven't hugged me." It is worth noting that his comment was met with widespread applause and agreement by the other group members with another participant adding, "I just feel at home when I come here." [Site C]

While not all providers feel comfortable showing physical affection for patients - and it may not be appropriate to do so with patients who have histories of physical or sexual trauma - it is worth remembering that the provider's job permits them to touch their patients by shaking hands and conducting physical examinations. Moreover, for some participants, physical touch was not only a way for their provider to demonstrate care and acceptance but also a fundamental part of the provider's job.

When I met [my doctor], he was a completely different man.

He knew I was a diabetic, so he had that protocol to check the bottoms of my feet to make sure I didn't have no open wounds, check around my testicles, check my breasts to make sure that there was no lumps or anything [...] He did it the first six years, and then the other eight years he just would call me in the office, you got an appointment. Okay, I come in there, first thing he's busy on the computer. He never tells me to lay down on the bed to check me. So that began to kind of bother me. [Site A]

These findings suggest that patients may benefit from "the laying on of hands" by their providers, whether to give them a hug or conduct a physical exam. Benefits range from the emotional solace patients get from knowing that they are accepted and cared for to the simple reassurance of knowing that providers are doing their jobs. 


\section{Engagement is a two-way street: "I need to be a part of that process."}

The majority of participants expressed that it was important to work in partnership with their providers rather than "just sitting back and saying, yes, Doctor, no, Doctor, whatever you say, Doctor." [Site C]. Some participants described the process of working collaboratively in explicit terms, including tailoring their treatment to their specific goals and engaging in discussions with their providers about treatment options:

With my doctor, I know when I first met her, I'd just had my son, and what we did, we sat down and she had me make a list of everything I wanted to accomplish. So it's not - she didn't throw anything on me. She suggested. But at the end it was me. I had to do it. [Site A]

Don't expect me as a patient to just accept, because you're a doctor, that everything that comes out of your mouth is law. I need to be part of that process. [Site B]

Whereas many participants outlined collaboration in positive terms, other participants discussed how this quality was lacking in their relationships with providers, which they framed as incompatibility. Some participants complained of clinic policies that limited their ability to change providers, putting them in the uncomfortable position of remaining with a provider to whom they did not feel connected. One participant summarized:

If a person isn't compatible with the caregiver, the management of your care goes downhill [...] if you can't work together, then you can't get what it is that you need. If you can't work with the doctor that you're assigned, how do you go about getting a different one? Why is it so hard to get a different one? [Site B]

Later, another participant shared a story of requesting a change in providers because he "had a doctor that wanted to do it his way." This participant described how he was "totally honest with [his provider]" and told him that their relationship wasn't going to work for him because "there was no connection." [Site B]

While some patients may feel empowered enough to advocate for themselves and switch from providers with whom they are incompatible, other participants described having to "train" their providers to understand and respect their perspective as the patient:

I've been with my provider for like 23, 24 years, and I trained her. You have to be able to go in there and tell them, no, I don't feel comfortable with this, and you have to be your own advocate. [Site B]

I've trained more doctors than I care to count. But it's a matter of whether or not that doctor is willing to do that. 'Cause the last thing I want is to go in and have a doctor tell me, you don't know what you're talking about, I'm the doctor, you're the patient. Well, excuse me, I've lived in my skin for 43 years, I know what I'm talkin' about, okay? [Site B]

In these cases, it is worth noting that the onus was on the patients to engage the provider in a dialog about care, rather than on the provider to elicit their patients' thoughts and feelings about treatment.

Given the significant power differential inherent in the patient-provider relationship, it should ultimately be the provider's responsibility to model this type of cooperation. For example, one participant described how he and his provider collaborated this way:

Anything that I wanted to do, she would make suggestions. It wasn't - you need to take this medication, you need to do this - she sat down and she listened to me. There could be times that we be sittin' here and I could be talkin' and she'd just sit there and just look at me. But she knows that I need to say some more, and we'd just sit there and kinda just look at each other for maybe a minute or two, and everything else just starts spillin' out. [Site A]

In this case, the provider took specific steps to make the relationship more egalitarian by making suggestions, rather than giving orders, and attuning to the needs of her patient by giving him more time to talk before she offered her thoughts or opinions.

The above quote exemplifies the collaborative nature of a strong patient-provider relationship. Similarly, another participant viewed engagement as a two-way street and her provider's ability and willingness to commit to building a strong and intimate relationship were fundamental to her engagement:

I'm just saying, I need to be engaged in something, it's like - a marriage. To me, it's like [...] I want to be with you the rest of my life - to me that is an engagement. Not that he wants to marry me, but he really wants to be a close friend. [Site A]

Interestingly, the theme of "provider as friend" appeared in several other focus groups, with some participants describing 
their providers as their "best friend" and others also invoking the language of marriage vows:

To me it's [...] if you don't trust who you're seein', and them knowin' what's in your best interest, I don't know where I'd be without my doctor. My doctor's like my best friend. [Site A]

It's more than doctor-patient relationship. It's a friendship, because if you look at it, if that person doesn't go - you're basically gonna be in that person's life for the rest of your life. And it's like - til death do we part, and you're putting your life into this person's hands. [Site A]

She's like part of my friggin' life, man. Part of my life. [Site C]

The line between provider and friend became blurred when patients perceived their providers were making a sincere effort to build a strong relationship. Despite its unique characteristics, the patient-provider relationship is at its core a human relationship, and as a result, many of the same qualities essential to establishing and maintaining a strong connection in other relationships, like close friendships and marriages, also apply to the patient-provider relationship.

\section{Discussion}

The themes that emerged showed similarities between the patient-provider relationship and the therapeutic alliance for the promotion of better engagement in HIV care. Nearly all participants agreed that having a trusting, nonjudgmental and collaborative patient-provider relationship was essential to their engagement in care. These qualities are also found in strong therapeutic alliances. ${ }^{23-26}$ Patients are more likely to continue psychotherapy if they feel they have a strong working relationship with their therapist, whereas a weak therapeutic alliance may lead patients to terminate psychotherapy prematurely. ${ }^{11,12}$ Similarly, our findings demonstrate that a strong patient-provider relationship likely facilitated better appointment attendance and adherence to provider recommendations.

Additionally, trust and intimacy are necessary in both kind of relationships. Effective care necessitates that patient be forthright about aspects of their life that may impact their health and treatment, including sensitive information concerning substance use and sexual behaviors. Providers who respond to the disclosure of sensitive information in an understanding and nonjudgmental manner reduce the chance of having what they say "go in one ear and out the other." When patients do not share sensitive information or allow themselves to be vulnerable, and when providers do not validate and attend to the vulnerability their patients are experiencing, it is likely to have a negative effect on the patient-provider relationship and quality of care the patients receive.

Although it seems self-evident that patients would be more likely to engage in care if they feel better after leaving their HIV care appointments, among participants in the study, feeling better was not necessarily related to the biomedical treatment they received, but rather to the empathetic and caring atmosphere that providers and staff created by knowing their names, genuinely inquiring about them and their loved ones, treating them with respect and compassion, and touching them. Other research supports this conclusion: in one inner-city clinic, patients who reported that their provider knew them as a person were more likely to be adherent to HIV treatment and achieve viral suppression. ${ }^{27}$ More work is needed in this area, but our findings support the idea that purposeful efforts to create and promote such environments could be an effective intervention in and of itself.

Given that HIV stigma is maintained through interpersonal interactions and relationships that reinforce the social inequality between HIV negative and HIV positive people, ${ }^{28}$ one benefit of a strong patient-provider relationship may be its potential to mitigate the negative effects of stigma. Patients who feel that their providers treat them in an empathetic, accepting and authentic manner may have the corrective experience of being in a relationship free of damaging effects of HIV stigma. Moreover, providers who use their position to create a safe non-stigmatizing space for their patients may strengthen the patient-provider relationship and be more successful in helping patients engage in their care.

\section{Putting findings in the context of a therapeutic alliance}

Based on the affirming stories shared during the focus group discussions, some HIV care providers deliver care in a similar manner to therapists engaging in client-centered psychotherapy. According to client-centered therapy, certain core conditions must be present for constructive behavior change to occur. ${ }^{29}$ These conditions include congruence, unconditional positive regard and empathic understanding, which all issue from the provider and therefore do not rely on any outside factors or other interventions like medications.

Briefly, in the context of HIV care, congruence means that providers are genuine in their interactions with patients, and are willing and able to acknowledge and own their inner experience and feelings in an authentic way. This manifests with patients as a "relaxed openness," which allows the patient to 
realize over time that their provider is not making "covert diagnostic judgments or having reactions that are off-limits to the [patients]. ${ }^{29}$ Unconditional positive regard refers to providers having a respectful, appreciative and nonjudgmental stance toward their patients, which is not predicated on the patient's choices, opinions or behaviors. ${ }^{29}$ Empathic understanding is defined as the willingness and ability to enter and feel thoroughly at home in the perceptual world of another, while being sensitive from moment-to-moment to the changes in the other's felt experience and the meaning being assigned to it. ${ }^{30}$ As the word "core" suggests, these conditions are fundamental to establishing and maintaining a strong therapeutic alliance and, we would contend, a strong patient-provider relationship as well.

\section{Strategies/recommendations}

Practical strategies common to building strong therapeutic alliances were present in the patient narratives, including simple gestures like referring to patients by name, expressing a genuine interest in their lives, eliciting their thoughts and feelings about their treatment, and human touch. While physical affection may be viewed as taboo in a therapeutic context, medical providers are usually allowed to demonstrate care through physical touch, which some patients may consider to be a vital aspect of their care experience. While these strategies may appear easy to implement, we found that patients are acutely attuned to the nonverbal behaviors and actions of their providers; so, greater attention to the genuineness of how these strategies are enacted can improve their effectiveness.

Additional therapeutic practices that could also be adopted by medical providers include the concepts of "process conversations" and "rupture and repair." At the start of treatment, therapists often engage patients in a dialog about the process of therapy by; for example, asking what qualities the patients are looking for in a therapist and exploring any prior positive or negative experiences the patients have had with therapy. Process conversations are a useful way to elicit values and expectations a patient may hold, but is unable to clearly articulate, and allow the therapist to tailor their approach accordingly.

Moreover, process conversations are a useful strategy to prevent tension or breakdown in the collaborative relationship, commonly referred to as alliance "ruptures" in psychotherapy. ${ }^{31}$ However, unlike therapists, providers may not receive extensive training on how to build and maintain strong working relationships. In addition, medical providers, generally speaking, do not have as much time to spend with their patients as psychotherapists. As a result, providers may not notice when ruptures have occurred or have the opportunity to repair them when they do. If unaddressed, these ruptures can irreparably damage the relationship, as happened with the participant who "had a doctor that wanted to do it his way." However, if providers repair ruptures with patients when or soon after they occur, they may find that the relationship is stronger afterward because ruptures offer the opportunity for deeper intimacy and trust in the relationship.

The patient-provider relationship is not egalitarian, as providers often have unilateral control over what treatment and care patients receive. A provider who makes genuine attempts to repair any ruptures can help correct this imbalance because these strategies help ensure patients are receiving personalized care, while simultaneously conveying to patients that both they and their provider have a role in shaping and maintaining the relationship. Process conversations may be especially helpful in laying the foundation of trust for patients who have preconceived notions of what providers are like (ie, "quacks") or who may be suspicious of being "put in the line of fire." Providers who can acknowledge these beliefs and validate concerns may be more likely to "earn" trust.

Based on our findings, additional suggestions include creating a welcoming reception and waiting area, offering providers in-depth training on how to build and maintain collaborative relationships with patients, allotting more time for medical appointments, thoughtful matching of new patients with providers and creating systems for patients to give confidential feedback to providers and to the clinic.

\section{Limitations}

While our sample was diverse with regard to race, ethnicity, gender and sexual orientation, there were only 43 participants. Moreover, focus groups were only conducted with patients in San Francisco, Seattle and Birmingham. As a result, it is unclear how patient preferences with regard to HIV care and, more specifically, the patient-provider relationship might vary beyond our sample and in other geographic areas of the USA with varying cultural norms and access to health care.

Furthermore, these findings are an exploratory look at the similarities between the patient-provider relationship in HIV care and the therapeutic alliance in psychotherapy, with a focus on patient narratives detailing the impact of the patient-provider relationship on engagement in care. Our sample was not purposefully recruited based on the therapeutic alliance. So, further qualitative and quantitative 
investigation is necessary to determine whether the therapeutic alliance in HIV care is associated with treatment adherence and specific outcomes, including appointment attendance and virologic suppression.

Limitations of applying a therapeutic framework to HIV care include differences in the structure and format of psychotherapy compared to HIV care settings. During the past 3 decades, the patient-provider relationship has become a secondary concern in HIV care as antiretroviral treatments have become the central, if not single, intervention seen as vital to the health of PLWH. As a result, HIV providers often confront significant barriers to building strong working relationships with their patients, including clinic policies that limit the frequency with which they are able to see and the time providers are able to spend with their patients. Psychotherapists, on the other hand, often have the luxury of seeing their patients for nearly an hour at a time on a weekly basis. Thus, they have ample opportunities to build strong working relationships, engage in process conversations and repair ruptures. While many providers do provide compassionate care despite these challenges, more research is needed to determine what clinics and policy-makers can do to support providers' efforts to build strong and enduring working relationships with patients.

\section{Conclusion}

We identified a variety of factors that can promote or impede engagement in care. We used the concept of the therapeutic alliance to highlight certain relationship factors that facilitated engagement, while offering strategies to help providers build and maintain strong relationships with patients. Most participants thought a strong patient-provider relationship characterized by trust, intimacy and collaboration was an important aspect of their care experience. These data suggest that HIV treatment is not synonymous with HIV care because as our focus group participants made clear - treatment can be provided in the absence of a caring atmosphere or a strong patient-provider relationship. We contend that a strong patient-provider relationship is crucial for patients to feel cared for during clinical encounters, which can promote long-term and sustained engagement in HIV care.

\section{Disclosure}

The authors report no conflicts of interest in this work.

\section{References}

1. Ciechanowski PS, Katon WJ, Russo JE, Walker EA. The patient-provider relationship: attachment theory and adherence to treatment in diabetes. Am J Psychiatry. 2001;158(1):29-35.
2. Langst G, Seidling HM, Stutzle M, et al. Factors associated with medication information in diabetes care: differences in perceptions between patients and health care professionals. Patient Prefer Adherence. 2015;9:1431-1441.

3. Lederer S, Fischer MJ, Gordon HS, Wadhwa A, Popli S, Gordon EJ. Barriers to effective communication between veterans with chronic kidney disease and their healthcare providers. Clin Kidney J. 2015;8(6):766-771.

4. Rachlis B, Naanyu V, Wachira J, et al. Identifying common barriers and facilitators to linkage and retention in chronic disease care in western Kenya. BMC Public Health. 2016;16:741.

5. Rogal SS, Arnold RM, Chapko M, et al. The patient-provider relationship is associated with hepatitis $\mathrm{C}$ treatment eligibility: a Prospective Mixed-Methods Cohort Study. PLoS One. 2016;11(2):e0148596.

6. Song L, Weaver MA, Chen RC, et al. Associations between patientprovider communication and socio-cultural factors in prostate cancer patients: a cross-sectional evaluation of racial differences. Patient Educ Couns. 2014;97(3):339-346.

7. Camlin CS, Neilands TB, Odeny TA, et al; East AfricaInternational Epidemiologic Databases to Evaluate AIDS (EA-IeDEA) Consortium. Patient-reported factors associated with reengagement among HIV-infected patients disengaged from care in East Africa. AIDS. 2016;30(3):495-502.

8. Flickinger TE, Saha S, Moore RD, Beach MC. Higher quality communication and relationships are associated with improved patient engagement in HIV care. J Acquir Immune Defic Syndr. 2013;63(3): 362-366.

9. Summers RF, Barber JP. Therapeutic alliance as a measurable psychotherapy skill. Acad Psychiatry. 2003;27(3):160-165.

10. Higgins T, Larson E, Schnall R. Unraveling the meaning of patient engagement: a concept analysis. Patient Educ Couns. 2017;100(1): $30-36$.

11. Kokotovic AM, Tracey TJ. Working alliance in the early phase of counseling. J Couns Psychol. 1990;37(1):16-21.

12. Frank AF, Gunderson JG. The role of the therapeutic alliance in the treatment of schizophrenia: relationship to course and outcome. Arch Gen Psychiatry. 1990;47(3):228-236.

13. Sly R, Morgan JF, Mountford VA, Lacey JH. Predicting premature termination of hospitalised treatment for anorexia nervosa: the roles of therapeutic alliance, motivation, and behaviour change. Eat Behav. 2013;14(2):119-123.

14. Lambert MJ, Barley DE. Research summary on the therapeutic relationship and psychotherapy outcome. Psychother Theory Res Pract Train. 2001;38(4):357-361.

15. Greenberg LS, Watson JC, Elliot R, Bohart AC. Empathy. Psychother Theory Res Pract Train. 2001;38(4):380-384.

16. Monaghan M, Hilliard M, Sweenie R, Riekert K. Transition readiness in adolescents and emerging adults with diabetes: the role of patient-provider communication. Curr Diab Rep. 2013;13(6): 900-908.

17. Johnson MO, Chesney MA, Goldstein RB, et al. Positive provider interactions, adherence self-efficacy, and adherence to antiretroviral medications among HIV-infected adults: a mediation model. AIDS Patient Care STDs. 2006;20(4):258-268.

18. Dawson-Rose C, Cuca YP, Webel AR, et al. Building trust and relationships between patients and providers: an essential complement to Health Literacy in HIV Care. J Assoc Nurses AIDS Care. 2016; 27(5):574-584.

19. Johnson MO, Koester KA, Wood T, Neilands TB, Pomeranz JL, Christopoulos KA. Development of an Index of Engagement in HIV Care: an Adapted Internet-Based Delphi Process. JMIR Res Protoc. 2017;6(12):e224.

20. Mugavero MJ, Amico KR, Horn T, Thompson MA. The state of engagement in HIV care in the United States: from cascade to continuum to control. Clin Infect Dis. 2013;57(8):1164-1171. 
21. Thompson MA, Mugavero MJ, Amico KR, et al. Guidelines for improving entry into and retention in care and antiretroviral adherence for persons with HIV: evidence-based recommendations from an International Association of Physicians in AIDS Care panel. Ann Intern Med. 2012;156(11):817-833.

22. Hsieh HF, Shannon SE. Three approaches to qualitative content analysis. Qual Health Res. 2005;15(9):1277-1288.

23. Clemence AJ, Hilsenroth MJ, Ackerman SJ, Strassle CG, Handler L. Facets of the therapeutic alliance and perceived progress in psychotherapy: relationship between patient and therapist perspectives. Clin Psychol Psychother. 2005;12(6):443-454.

24. Farber BA, Doolin EM. Positive regard. Psychotherapy (Chic). 2011; 48(1):58-64.

25. Rogers CR. The necessary and sufficient conditions of therapeutic personality change 1957. J Consult Clin Psychol. 1992;60(6):827-832.
26. Horvath AO, Luborsky L. The role of the therapeutic alliance in psychotherapy. J Consult Clin Psychol. 1993;61(4):561-573.

27. Beach MC, Keruly J, Moore RD. Is the quality of the patient-provider relationship associated with better adherence and health outcomes for patients with HIV? J Gen Intern Med. 2006;21(6):661-665.

28. Earnshaw VA, Chaudoir SR. From conceptualizing to measuring HIV stigma: a review of HIV stigma mechanism measures. AIDS Behav. 2009;13(6):1160-1177.

29. Rogers CR. Client-centered therapy. Curr Psychother. 2013:95-150.

30. Rogers CR. Empathic: an unappreciated way of being. Couns Psychol. 1975;5(2):2-10

31. Safran JD, Muran JC, Eubanks-Carter C. Repairing alliance ruptures. Psychotherapy (Chic). 2011;48(1):80-87.

\section{Publish your work in this journal}

Patient Preference and Adherence is an international, peer-reviewed, open access journal that focuses on the growing importance of patient preference and adherence throughout the therapeutic continuum. Patien satisfaction, acceptability, quality of life, compliance, persistence and their role in developing new therapeutic modalities and compounds to optimize clinical outcomes for existing disease states are major areas of interest for the journal. This journal has been accepted for indexing on PubMed Central. The manuscript management system is completely online and includes a very quick and fair peer-review system, which is all easy to use. Visit http://www. dovepress.com/testimonials.php to read real quotes from published authors.

Submit your manuscript here: http://www.dovepress.com/patient-preference-and-adherence-journal 